\title{
THE ELECTROMAGNETIC GROUP: BOSONIC BRST CHARGE
}

\author{
V. ALDAYA ${ }^{a}$, R. LOLL ${ }^{b}$ and J. NAVARRO-SALAS ${ }^{a}$ \\ a Departamento de Fisica Teorica, Facultad de Físicas, Universidad de Valencia, E-46100 Burjasot (Valencia), Spain \\ and IFIC (Centro Mixto Universidad de Valencia-CSIC), E-46100 Burjasot (Valencia), Spain \\ b The Blackett Laboratory, Imperial College, London SW7 2BY, LiK
}

Received 10 July 1989

\begin{abstract}
We give an infinite-dimensional Lie group from which a group approach to quantization (GAQ) derives a Gupta-Bleuler-like quantization for the electromagnetic field. The incorporation into the group law of the gauge transformation properties of $A_{\mu}(x)$, $A_{\mu}(x) \rightarrow \Lambda_{\mu}(x)+\partial_{\mu} \phi$, requires a non-conventional generator which is related to the BRST charge.
\end{abstract}

In previous papers [1,2] we have analyzed the problem of quantizing constrained physical systems on the grounds of a group approach to quantization (GAQ) for which the usual $U$ (1) phase invariance of quantum mechanics is replaced with a bigger (super)group $\mathrm{T}$ containing a group of constraints $\mathrm{K}$. From the mathematical point of view $\mathrm{T}$ is the structure group of a principal bundle endowed with a Lic (super)group law, $\widetilde{\mathrm{G}}$, denominated quantum group $[1,2]$. The quantum constraints on the wave functions then appear as a natural generalization of the $U(1)$ equivariance that results in the $U(1)$ phase invariance. A particularly interesting case is that for which $\mathrm{T}$, or rather its Lie algebra, is a generalization of the BRST algebra given by Bowick and Gürsey [3]. The GAQ then provides the entire algebraic structure underlying BRST quantization.

The GAQ idea is to perform the quantization starting with the complex functions $\mathscr{F}$ on the "symmetry group" rather than the phase space. A naive quantization (prequantization or Bohr quantization) is achieved from the right generators acting on $\mathscr{F}$. This representation is highly reducible, and it is for that reason that a proper quantization mechanism must be followed. One can in fact check that there are nontrivial operators, the left generators $\tilde{X}^{\mathrm{L}}$ indeed, which commute with the representation $\tilde{X}^{\mathrm{R}}\left(\left[\tilde{X}^{\mathrm{L}}, \tilde{X}^{\mathrm{R}}\right]=0\right.$

* Research partially supported by the Comision Interministerial de Ciencia y Tecnologia (CICZT). is a general property for any Lic group). Then, reducing the representation means looking for a maximal set of compatible conditions on $\mathscr{F}$ trivializing the effect of left generators. For instance, the U(1) generator (of phase invariance in quantum mechanics) $\tilde{X}_{\zeta}^{\mathrm{R}} \equiv \Xi$ can be made trivial by imposing the so-called $\mathrm{U}(1)$ equivariance condition, $\Xi \psi=\mathrm{i} \psi$. The next step is to impose the maximum number of compatible conditions of the form $\tilde{X}^{\mathrm{L}} \psi=0$. This is accomplished by means of a polarization.

The usefulness of GAQ in quantizing field theories lies in the way the normal ordering comes out from the general scheme (it appears automatically from the choice of a particular subalgebra, the polarization subalgebra) and also in the underlying associative structure defining (super)Jacobi identities among all the operators entering the theory. Together, thesc properties avoid many infinities which are inherent in the standard quantization techniques.

Our aim in this letter is to present a framework based on GAQ constituting a first step toward the non-perturbative quantization of non-abelian gauge theories. The crucial point is to close a group containing among its parameters (apart from those of the Poincaré group) the Fourier coefficients of the gauge potential and the parameters of the gauge transformations with a proper action of the latter on the former. Although the structure of the mentioned group in the general non-abelian case is not known yet, the 
abelian one is rather feasible and we believe that it could provide the key to generalization.

We then find the simplest infinite-dimensional Lic group (the "electromagnetic group" $\widetilde{G}_{E}$ ) containing in a non-trivial way the Poincare group, the group generated by the Fock operators for a free electromagnetic field and the local $U(1)$ gauge group. As a quantum group, the structure group $T$ contains the centrally extended local $U(1)$ symmetry. Lie groups containing the Poincare group as well as the group generated by the Fock operators for a free field have already been considered and the corresponding quantum dynamics have been obtained from just the group manifold [4]. In particular a quantum group for the Proca field, $\widetilde{\mathrm{G}}_{\text {Proca }}$, was provided. In that case the transversality condition $\partial^{\mu} \Psi_{\mu}=0$ came from what in GAQ is the standard set of equations of motion, i.e., the charactcristic subalgebra $\mathscr{G}_{6}$ generating the kernel of the quantization form $\theta$ (see ref. [1] and references therein ) or, what is the same, the kernel of the Lie algebra cocycle of the $U(1)$ centrally extended group $\tilde{\mathrm{G}}_{\text {Proca }} \rightarrow \tilde{\mathrm{G}}_{\text {proca }} / \mathrm{U}(1)$.

The symmetry of the free electromagnetic field is, however, much more involved because of the nontrivial action of the local $U(1)$ gauge group (the structure group of our requested quantum group) on the electromagnetic field operators. Moreover, finding the composition law for a symmetry group is in general much more difficult than simply giving the action of it on the corresponding physical objects. This action must be of course recovered as an orbit of the group law, i.e. of the action of the group on itself. In our case (and disregarding for the time being the semidirect action of the Poincare group) the group law must account for the usual gauge transformation properties of $A_{\mu}, A_{\mu}(k) \rightarrow A_{\mu}(k)-\mathrm{i} k_{\mu} \phi(k)\left[A_{\mu}(x) \rightarrow\right.$ $A_{\mu}(x)+\partial_{\mu} \phi(x)$ in configuration space], as well as the "translations" by the Fock generators $a_{\mu}(k)$, $A_{\mu}(k) \rightarrow A_{\mu}(k)+a_{\mu}(k)$, that is to say,

$A_{\mu}(k)^{\prime} \rightarrow A_{\mu}(k)-\mathrm{i} k_{\mu} \phi(k)+a_{\mu}(k)$.

In order to motivate the composition law (see below) which must account for (1a) (at least to the lowest order), let us go to the simpler example of the action of the Poincare group $(\kappa, A)$ on space-time $(x)$ :

$x^{t}=\kappa+A x$.
The transformation ( $2 a)$ is associated with the following group law for the translation parameter:

$\kappa^{\prime \prime}=\kappa^{\prime}+\Lambda^{\prime} \kappa$,

(to be complemented with $A^{\prime \prime}=A^{\prime} A$ ) that allows us to interpret $\kappa$ as the space-time parameter itself. Indeed, $(2 a)$ is obtained from ( $2 b$ ) by just considering the unprimed parameters $(\kappa)$ as points and the primed ones ( $\kappa^{\prime}$ and $A^{\prime}$ ) as group parameters. Going back to the electromagnetic case, we identify the Fock variables $A(k)$ and the group parameters $a(k)$ writing the analog to $(2 \mathrm{~b})$,

$a_{\mu}(k)^{\prime \prime}=a_{\mu}(k)-\mathrm{i} k_{\mu l} \phi(k)^{\prime}+a_{\mu}(k)^{\prime}$,

but unlike eq. ( $2 b)$, where the action of the Lorentz subgroup on $\kappa$ is of a very special type (semidirect action), it is not yet a group law because the group law for a certain parameter cannot be linear in any other. That necessarily means introducing a new parameter, multiplying $\phi\left(k^{\prime}\right)$ in (1b), which will provide a non-trivial commutator among its associated generator in the Lie algebra and the one associated with the parameter $a_{\mu}(k)$.

We now write the simplest group law in Fock space compatible with the action (1a), which now appears as the first order of an orbit inside the group, and providing the fundamentals of the physical theory. On this group we make all calculations and at the end we add the semidirect action of the Poincare group, which does not modify the solutions and only contributes by simply providing new Noether invariants as a function on the basic operators $a_{\mu}(k), a_{\nu}^{\dagger}(k)$. We also discuss the connection with BRST symmetries where the role of BRST charge [5] is played by a fermionic version of the additional parameter here required.

Introducing the extra (real) parameter $b$ we propose the following group law for the electromagnetic field:

$$
\begin{aligned}
& a_{\mu}^{\prime \prime}(k)=a_{\mu}^{\prime}(k)+a_{\mu}(k)-\mathrm{i} k_{\mu} \phi^{\prime}(k) b \\
& \quad-\frac{1}{2 !} k_{\mu} k^{\nu} a_{\nu}^{\prime}(k) b^{2}, \\
& a_{\mu}^{\prime \prime \dagger}(k)=a_{\mu}^{\prime \dagger}(k)+a_{\mu}^{\dagger}(k)+\mathrm{i} k_{\mu} \phi^{\prime \dagger}(k) b \\
& \quad-\frac{1}{2 !} k_{\mu} k^{\nu} a_{\nu}^{\dagger}(k) b^{2},
\end{aligned}
$$




$$
\begin{aligned}
& \phi^{\prime \prime}(k)=\phi^{\prime}(k)+\phi(k)-\mathrm{i} k^{\mu} a_{\mu}^{\prime}(k) b, \\
& \phi^{\prime \prime+}(k)=\phi^{\prime \dagger}(k)+\phi^{\dagger}(k)+\mathrm{i} k^{\mu} a_{\mu}^{\prime \dagger}(k), \\
& b^{\prime \prime}=b^{\prime}+b \\
& \zeta^{\prime \prime}=\zeta^{\prime} \zeta \exp \left(-\frac{1}{2} \int \mathrm{d} \Omega_{k}\left(g ^ { \mu \nu } \left\{\left[a_{\mu}^{+\prime}(k)\right.\right.\right.\right. \\
& \left.\quad+\mathrm{i} k_{\mu} \phi^{\prime+}(k) b-\frac{1}{2} k_{\mu} k^{\sigma} a_{\sigma}^{\prime+}(k) b^{2}\right] a_{\nu}(k) \\
& \left.\quad-a_{\mu}^{\dagger}(k)\left[a_{\nu}^{\prime}(k)-\mathrm{i} k_{\mu} \phi^{\prime}(k) b-\frac{1}{2} k_{\mu} k^{\sigma} a_{\sigma}^{\prime}(k) b^{2}\right]\right\} \\
& \quad+\left\{\left[\phi^{+\prime}(k)+\mathrm{i} k^{\mu} a_{\mu}^{\prime \dagger}(k) b\right] \phi(k)\right. \\
& \left.\left.\left.\quad-\phi^{\dagger}(k)\left[\phi^{\prime}(k)-\mathrm{i} k^{\mu} a_{\mu}^{\prime}(k) b\right]\right\}\right)\right) .
\end{aligned}
$$

The group law (3) corresponds to a central extension by $U(1)$, parametrized by $i \ln \zeta$, of the group parametrized by the rest of the parameters.

From (3) we derive the left and right generators:

$$
\begin{aligned}
& \tilde{X}_{a_{\mu}(k)}^{\mathrm{L}}=\frac{\partial}{\partial a_{\mu}(k)}+\frac{1}{2} \mathrm{i} a^{\dagger \mu}(k) \Xi, \\
& \tilde{X}_{a_{\mu}^{\dagger}(k)}^{\mathrm{L}_{\dagger}}=\frac{\partial}{\partial a_{\mu}^{\dagger}(k)}-\frac{1}{2} \mathrm{i} a^{\mu}(k) \Xi, \\
& \tilde{X}_{b}^{\mathrm{L}}=\frac{\partial}{\partial b}-\mathrm{i} k_{\mu} \phi(k) \frac{\partial}{\partial a_{\mu}(k)}+\mathrm{i} k_{\mu} \phi^{\dagger}(k) \frac{\partial}{\partial a_{\mu}^{\dagger}(k)} \\
& -\mathrm{i} k^{\mu} a_{\mu}(k) \frac{\partial}{\partial \phi(k)}+\mathrm{i} k^{\mu} a_{\mu}^{\dagger}(k) \frac{\partial}{\partial \phi^{\dagger}(k)}, \\
& \tilde{X}_{\phi^{\prime}(k)}^{\mathrm{L}}=\frac{\partial}{\partial \phi(k)}+\frac{1}{2} \mathrm{i} \phi(k) \Xi, \\
& \tilde{X}_{\phi^{\dagger}(k)}^{\mathrm{L}}=\frac{\partial}{\partial \phi^{\dagger}(k)}-\frac{1}{2} \mathrm{i} \phi(k) \Xi, \quad \tilde{X}_{\zeta}^{\mathrm{L}}=\mathrm{i} \zeta \frac{\partial}{\partial \zeta} \equiv \Xi, \\
& \tilde{X}_{a_{\mu}(k)}^{\mathrm{R}}=\frac{\partial}{\partial a_{\mu}(k)}-\mathrm{i} k^{\mu} b \frac{\partial}{\partial \phi(k)}-\frac{1}{2} k^{\mu} k^{\sigma} b^{2} \frac{\partial}{\partial a^{\sigma}(k)} \\
& -\frac{1}{2} \mathrm{i}\left[a^{\dagger \mu}(k)-\mathrm{i} k^{\mu} \phi^{\dagger}(k) b-\frac{1}{2} k^{\mu} k^{\sigma} a_{\sigma}^{\dagger}(k) b^{2}\right] \Xi, \\
& \tilde{X}_{a_{\nu}^{\dagger}(k)}^{\mathrm{R}}=\left(\tilde{X}_{a_{\nu}(k)}^{\mathrm{R}}\right)^{+}, \quad \tilde{X}_{b}^{\mathrm{R}}=\frac{\partial}{\partial b}, \\
& \tilde{X}_{\phi(k)}^{\mathrm{R}}=\frac{\partial}{\partial \phi(k)}-\mathrm{i} k_{\mu} b \frac{\partial}{\partial a_{\mu}(k)} \\
& -\frac{1}{2} \mathrm{i}\left[\phi^{\dagger}(k)-\mathrm{i} k^{\mu} a_{\mu}^{\dagger}(k) b\right] \Xi, \\
& \tilde{X}_{\phi^{\dagger}(k)}^{\mathrm{R}}=\left(\tilde{X}_{\phi(k)}^{\mathrm{R}}\right)^{\dagger}, \quad \tilde{X}_{(\zeta)}^{\mathrm{R}}=\mathrm{i} \zeta \frac{\partial}{\partial \zeta} \equiv \Xi . \\
&
\end{aligned}
$$

The Lie algebra is easily obtained from either (4) or (5) giving the following non-zero commutators:

$$
\begin{aligned}
& {\left[\tilde{X}_{a_{\mu}^{\dagger}\left(k^{\prime}\right)}^{\mathrm{R}}, \tilde{X}_{a_{\nu}(k)}^{\mathrm{R}}\right]=g^{\mu \nu} \delta\left(k^{\prime}-k\right) \Xi,} \\
& {\left[\tilde{X}_{\phi^{\mathrm{R}}(k)}^{\mathrm{R}}, \tilde{X}_{b}^{\mathrm{R}}\right]=\mathrm{i} k_{\mu} \tilde{X}_{a_{\mu}(k)}^{\mathrm{R}},} \\
& {\left[\tilde{X}_{\phi^{\dagger}(k)}^{\mathrm{R}}, \tilde{X}_{b}^{\mathrm{R}}\right]=-\mathrm{i} k_{\mu^{\prime}} \tilde{X}_{a_{\mu}^{+}(k)}^{\mathrm{R}_{+}},} \\
& {\left[\tilde{X}_{a_{\mu}(k)}^{\mathrm{R}}, \tilde{X}_{b}^{\mathrm{R}}\right]=\mathrm{i} k^{\mu} \tilde{X}_{\phi^{\prime}(k)}^{\mathrm{R}},} \\
& {\left[\tilde{X}_{a_{\mu}^{\dagger}(k)}^{\mathrm{R}}, \tilde{X}_{b}^{\mathrm{R}}\right]=-i k^{\mu} \tilde{X}_{\phi^{\dagger}(k)}^{\mathrm{R}},} \\
& {\left[\tilde{X}_{\phi^{\dagger}(k \dagger)}^{\mathrm{R}}, \tilde{X}_{\phi(k)}^{\mathrm{R}}\right]=\delta\left(k^{\prime}-k\right) \Xi .}
\end{aligned}
$$

Now we can compare that part of the Lie (group) cocycle involving the vector parameters $a_{\mu}^{\dagger}\left(k^{\prime}\right)$ and $a_{\nu}(k)$ with the cocycle on the Proca group, $\mathrm{G}_{\text {Proca }}$, i.e., the cocycle for massive fields. For the massive field, which is not gauge invariant, the Lie algebra cocycle is $g^{\mu \nu}-k^{\mu} k^{\nu} / m^{2}$, where $m$ is the mass of the vector particle. In the present massless case the term in the cocycle proportional to $k^{\mu} k^{\nu}$ is forbidden by the Jacobi identity among $\phi(k), a_{\mu}^{\dagger}\left(k^{\prime}\right)$ and $b$ or $\phi^{\dagger}\left(k^{\prime}\right)$, $a_{\mu}\left(k^{\prime}\right)$ and $b$. In other words, the massive term is forbidden by the gauge symmetry. However, the electromagnetic symmetry ( 3 ) could be written off shell, a fact which is important if we wish to include the interaction with fermions in the future.

Rather than the $U(1)$ central extension structure of (3), the quantum group structure we are interested in is that of a principal bundle on the "phase space" manifold $\widetilde{G}_{E} / T$ with structure group $T$ generated by what we could call unphysical operators, i.e. $\left\{\phi^{\dagger}(k), \phi\left(k^{\prime}\right), b, \zeta\right\}$ as well as the "longitudinal" components of the Fock operators which are required to close on the group T. The structure group generators will act as constraints of the physical states through the $T$ equivariance condition [1] generalizing the traditional $U(1)$ phase invariance in quantum mechanics ( the infinitesimal version of which is $\left.\tilde{X}_{\zeta}^{\mathrm{R}} \psi=\mathrm{i} \psi\right)$. The Lie algebra $\mathscr{T}$ of the structure group $\mathrm{T}$ is (in terms of right generators)

$$
\mathscr{T}=\left\langle k^{\mu} \tilde{X}_{a_{\mu}^{\dagger}(k)}^{\mathrm{R}_{t}}, k^{\nu} \tilde{X}_{a_{\nu}(k)}^{\mathrm{R}}, \tilde{X}_{\phi^{\dagger}(k)}^{\mathrm{R}}, \tilde{X}_{\phi(k)}^{\mathrm{R}}, \widetilde{X}_{b}^{\mathrm{R}}, \tilde{X}_{\zeta}^{\mathrm{R}}\right\rangle .
$$

Another important subalgebra of $\widetilde{\mathrm{G}}_{\mathrm{E}}$ is the characteristic subalgebra, i.c., the kernel of the left-algebra cocycle:

$\mathscr{G}_{\mathscr{K}^{\prime}}=\left\langle\tilde{X}_{b}^{\mathrm{L}}\right\rangle$. 
$\mathscr{G}_{6}$ is generated by those operators which are not canonically conjugated to any other in the whole group and permit the unambiguous definition of a polarization. We must remark that all the Poincare generators would be included in $\mathscr{G}_{\mathscr{G}}$ had we considered this symmetry explicitly from the beginning.

A full polarization $\mathscr{P}$ is a maximal left subalgebra containing $\mathscr{G}_{\mathscr{G}}$ and excluding the central generator $\tilde{X}_{\zeta}^{\mathrm{L}}$ (to prevent pairs of canonically conjugate variables occurring in $\mathscr{P}$ ). In our case we find

$\mathscr{P}=\left\langle\tilde{X}_{b}^{\mathrm{L}}, \tilde{X}_{a_{\mu}^{\dagger}(k)}^{\mathbf{L}_{1}}, \tilde{X}_{\phi^{\dagger}(k)}^{\mathrm{L}}\right\rangle$.

The quantization mechanism takes place as follows. We start with the complex functions on $\bar{G}_{E}$ satisfying the ordinary $U(1)$ equivariance condition

$\tilde{X}_{\zeta}^{\mathrm{R}} \psi=\mathrm{i} \psi$

as in the case of a non-constrained theory. We now impose the polarization conditions on $\psi$,

$\tilde{X}^{\mathrm{L}} \psi=0 \quad \forall \tilde{X}^{\mathrm{L}} \in \mathscr{P}$,

which include, in general, the generalized quantum equations of motion. Those steps suffice for the quantization of an unconstrained theory as for instance that of the Proca field whose transversality condition comes from the polarization conditions [4]. The integration of (11) is easily achieved:

$$
\begin{aligned}
& \tilde{X}_{a_{\mu}^{\dagger}(k)}^{\mathrm{L}_{1}} \psi=0=\tilde{X}_{\phi^{\dagger}(k)}^{\mathrm{L}} \psi \\
& \Rightarrow \psi=\zeta \exp \left(-\frac{1}{2} \int \mathrm{d} \Omega a_{\mu}^{\dagger} a^{\mu}\right) \exp \left(-\frac{1}{2} \int \mathrm{d} \Omega \phi^{\dagger} \phi\right) \\
& \times \Phi\left(a_{\nu}(k), \phi(k), b\right),
\end{aligned}
$$

where $\Phi$ is an arbitrary function, and

$$
\begin{aligned}
& \tilde{X}_{(b)}^{\mathrm{L}} \psi=0 \\
& \quad \Rightarrow \Phi=\varphi\left(a_{\mu}(k)+\mathrm{i} k_{\mu} \phi(k) b-\frac{1}{2} k_{\mu} k^{v} a_{\nu}(k) b^{2}\right) \\
& \quad \times f\left(\phi(k)+\mathrm{i} k^{\mu} a_{\mu}(k) b\right),
\end{aligned}
$$

with $\varphi$ and $f$ also arbitrary functions. The general solution can be decomposed into the following basic states:

$$
\begin{aligned}
& |0\rangle=\zeta \exp \left(-\frac{1}{2} \int \mathrm{d} \Omega a_{\mu}^{\dagger} a^{\mu}\right) \exp \left(-\frac{1}{2} \int \mathrm{d} \Omega \phi^{\dagger} \phi\right) \\
& \equiv W \quad \text { (the vacuum) },
\end{aligned}
$$

$$
\begin{aligned}
& \left|a_{\nu}(k)\right\rangle=W\left[a_{\nu}(k)+\mathrm{i} k_{\nu} \phi(k) b-\frac{1}{2} k_{\nu} k^{\mu} a_{\mu}(k) b^{2}\right], \\
& |\phi(k)\rangle=W\left[\phi(k)+\mathrm{i} k^{\mu} a_{\mu}(k) b\right] . \quad(14 \text { cont'd })
\end{aligned}
$$

For a constrained system we have to impose the rest of the equivariance conditions [1]

$$
\begin{aligned}
& \tilde{X}^{\mathrm{R}} \psi=D(X) \psi \\
& \quad \forall \tilde{X}^{\mathrm{R}} \in \mathscr{T}_{|0\rangle} \equiv\left\langle k^{\mu} \tilde{X}_{a_{\mu}}^{\mathrm{R}}, \tilde{X}_{\phi(k)}^{\mathrm{R}}, \tilde{X}_{(b)}^{\mathrm{R}}\right\rangle \subset \mathscr{T},
\end{aligned}
$$

where $D$ is a representation (characterizing the quantization) of $\mathscr{T}_{|0\rangle}$ which is the subalgebra of $\mathscr{T}$ annihilating the vacuum $|0\rangle . \mathscr{T}_{10\rangle}$ is, in general, the biggest subalgebra of $\mathscr{T}$ that can be imposed in (15). The use of the whole $\mathscr{T}$ in the equivariance condition causes the theory to be trivial or makes that equation incompatible if conjugate coordinate-momentum pairs like $\phi(k), \phi^{\dagger}(k)$ are present in $\mathscr{T}$. At most, those generators in $\mathscr{T}$ excluded from (15) can be employed to establish compatibly an equivalence relation among wave functions. In our present case, and taking the trivial representations $D=0$, the operator $\widetilde{X}_{\phi(k)}^{\mathrm{R}}$ annihilate $|0\rangle$ and $\left|a_{\nu}(k)\right\rangle$ and therefore it does not lead to non-trivial conditions. On the other hand, and because of $\widetilde{X}_{\phi(k)}^{\mathrm{R}}\left|\phi\left(k^{\prime}\right)\right\rangle=\delta\left(k^{\prime}-k\right)|0\rangle$ $\neq 0$, no $|\phi\rangle$ states are allowed as a consequence of (15). On the other hand, $\bar{X}_{b}^{\mathrm{R}}=\partial / \partial b$ acting on the linear combination $\varepsilon^{\mu}(k)\left|a_{\mu}(k)\right\rangle$ gives

$\tilde{X}_{b}^{\mathrm{R}} \varepsilon^{\mu}(k)\left|a_{\mu}(k)\right\rangle=\mathrm{i} \varepsilon^{\mu}(k) k_{\mu}|\phi(k)\rangle$,

and hence only those states $\varepsilon^{\mu}(k)\left|a_{\mu}(k)\right\rangle$ are physical for which

$\varepsilon^{\mu}(k) k_{\mu}=0$.

The condition $k_{\mu} \tilde{X}_{a_{\mu}(k)}^{\mathrm{R}} \psi=0$ does not impose any new restriction as can be observed from the commutator $\left[\widetilde{X}_{\phi(k)}^{\mathrm{R}}, \widetilde{X}_{b}^{\mathrm{R}}\right]=-\mathrm{i} k_{\mu} \widetilde{X}_{a_{\mu}(k)}^{\mathrm{R}}$. Finally, the operators $\widetilde{X}_{b}^{\mathrm{R}}, \widetilde{X}_{\phi^{\dagger}(k)}^{\mathrm{R}}$ and $k^{\mu} \tilde{X}_{a_{\mu}^{+}(k)}^{\mathrm{R}}$, which close on the right version of the algebra $\mathscr{P} \cap \mathscr{T}$, generate the spurious states. In particular, the states

$\tilde{X}_{b}^{\mathrm{R}}|\phi(k)\rangle=\mathrm{i} k^{\mu}\left|a_{\mu}(k)\right\rangle$

are spurious (zero norm) states. The same happens with $k^{\mu} \tilde{X}_{a_{\mu}^{\dagger}(k)}^{\mathrm{R}}|0\rangle=k^{\mu}\left|a_{\mu}(k)\right\rangle$. On the other hand, the operators $\widetilde{X}_{\phi^{+}(k)}^{\mathrm{K}}$ do not create physical states and hence no new spurious states are defined. This mechanism constitutes the group approach version of the Gupta-Bleuler quantization.

We now resume the results (16) in the following 
way. Physical states are polarized functions with "zero $\phi(k)$ number" annihilated by the operator $\widetilde{X}_{b}^{\mathbf{R}}$, modulus those states created by $\widetilde{X}_{b}^{R}$. This mechanism defines what we could call a $b$ cohomology.

The quantization could easily be completed with the addition of the Poincaré group and the computation of the quantization form, the Noether invariant, etc. Here we only give the prescription for including the Poincaré action. Apart from the composition law for itself, $x^{\prime \mu}=x^{\prime \mu}+\Lambda_{\nu}^{\prime \mu} x^{\nu}, A^{\prime \prime}=\Lambda^{\prime} A$, every unprimed vector parameter must be multiplied by $\Lambda^{\prime \prime}$, the primed parameters $a_{\mu}^{\prime}(k), \phi^{\prime}(k)$ by $\exp (-i k$. $\left.\Lambda^{\prime} x\right)$ and $a_{\mu}^{\prime \dagger}(k), \phi^{\prime \dagger}(k)$ by $\exp \left(\mathrm{i} k \cdot A^{\prime} x\right)$ (see the case of the Proca group in ref. [4]).

We can further clarify the role of the parameter $b$ which was required to close on the group. To this aim let us consider the transformation properties under the BRS charges $Q$ and $\bar{Q}$ of $A_{\mu}(x)$ as well as the ghost and anti-ghost fields $\phi^{*}(x), \phi(x)$ (see for instance the review of ref. [6]). They are

$\{Q, \phi(x)\}=-\mathrm{id}_{\mu} A^{\mu}(x), \quad\{\bar{Q}, \phi(x)\}=0$,

$\left\{Q, \phi^{*}(x)\right\}=0, \quad\left\{\bar{Q}, \phi^{*}(x)\right\}=\mathrm{id}_{\mu} A^{\mu}(x)$,

$\left[Q, A_{\mu}(x)\right]=-\mathrm{i} \partial_{\mu} \phi^{*}(x), \quad\left[\bar{Q}, A_{\mu}(x)\right]=\mathrm{i}_{\mu} \phi(x)$,

$\left[Q, \partial^{\mu} A_{\mu}(x)\right]=0, \quad\left[\bar{Q}, \partial^{\mu} A_{\mu}(x)\right]=0$.

Writing the Fourier decomposition of $A_{\mu}(x), \phi(x)$ and $\phi^{*}(x)$,

$$
\begin{aligned}
& A_{\mu}(x)=\int \mathrm{d} \Omega_{k}\left[a_{\mu}(k) \exp (-\mathrm{i} k \cdot x)\right. \\
& \left.\quad+a_{\mu}^{\dagger}(k) \exp (\mathrm{i} k \cdot x)\right], \\
& \phi(x)=\int \mathrm{d} \Omega_{k}[\phi(k) \exp (-\mathrm{i} k \cdot x) \\
& \left.\quad+\Psi^{\dagger}(k) \exp (\mathrm{i} k \cdot x)\right], \\
& \phi^{*}(x)=\int \mathrm{d} \Omega_{k}[\Psi(k) \exp (-\mathrm{i} k \cdot x) \\
& \left.\quad+\phi^{\dagger}(k) \exp (\mathrm{i} k \cdot x)\right\},
\end{aligned}
$$

we get the following superalgebra (we only write the non-zero terms):

$$
\begin{aligned}
& {\left[a_{\mu}^{\dagger}(k), a_{\nu}\left(k^{\prime}\right)\right]=g_{\mu \nu} \delta\left(k-k^{\prime}\right) \Xi,} \\
& {\left[a_{\mu}(k), Q\right]=k_{\mu} \Psi(k), \quad\left[a_{\mu}^{\dagger}(k), \bar{Q}\right]=k_{\mu} \Psi^{\dagger}(k),} \\
& {\left[a_{\mu}^{\dagger}(k), Q\right]=-k_{\mu} \phi^{\dagger}(k), \quad\left[a_{\mu}(k), \bar{Q}\right]=-k_{\mu} \phi(k),} \\
& \left\{\phi^{\dagger}(k), \phi\left(k^{\prime}\right)\right\}=\delta\left(k-k^{\prime}\right) \Xi,
\end{aligned}
$$

$$
\begin{aligned}
& \left\{\Psi^{\dagger}(k), \Psi\left(k^{\prime}\right)\right\}=\delta\left(k-k^{\prime}\right) \Xi, \\
& \{\phi(k), Q\}=k^{\mu} a_{\mu}(k), \quad\left\{\phi^{\dagger}(k), \bar{Q}\right\}=k^{\mu} a_{\mu}^{\dagger}(k), \\
& \left\{\Psi^{\dagger}(k), Q\right\}=-k^{\mu} a_{\mu}^{\dagger}(k), \quad\{\Psi(k), \bar{Q}\}=-k^{\mu} a_{\mu} .
\end{aligned}
$$

For this very special case of an abelian gauge theory (19) can be turned into a Lie algebra by simply replacing anticommutator with ordinary commutator. Then a simple change of basis

$b \equiv(Q+\bar{Q}) / \sqrt{2}, \quad \phi(k) \equiv[\Psi(k)-\phi(k)] / \sqrt{2}$,

$a_{\mu}(k) \equiv a_{\mu}(k), \quad a_{\mu}^{\dagger}(k) \equiv a_{\mu}^{\dagger}(k)$

identifies the algebra (6) as a subalgebra of the bosonized version of (19). A rcmarkable property of (19) and its bosonized analogue is that, unlike in the case of the algebra (6), the condition $k^{2}=0$ arises from the Jacobi identities [even before extending by the global U(1)].

We mention, finally, that obtaining a BRST symmetry was not our main purpose. Rather, we simply wanted to achieve the quantization on just a group $\left(\bar{G}_{E}\right)$ and by means of a method compatible with constraints. The symmetry $b$ then came out as a requirement of the theory. The connection with BRST symmetries must be interpreted in the sense that (super)groups with that sort of structure are strictly neccssary in a group approach to (exact) quantization of abelian and non-abelian gauge theories, although in the latter case a fermionic BRST symmetry might be required to close a quantum (super)group.

We thank Vicente Caselles for very valuable discussions.

\section{References}

[1] V. Aldaya, J. Navarro-Salas and A. Ramírcz, Commun. Math. Phys. 112 (1989) 541.

[2] V. Aldya, R. Loll and J. Navarro-Salas, Phys. Lett. B 225 (1989) 340 .

[3] M.J. Bowick and F. Gürsey, Phys. Lett. B 175 (1986) 182; Nucl. Phys. B 283 (1987) 331.

[4] V. Aldya, J.A. de Azcárraga and S. Garcia, J. Phys. A 21 (1988) 4265.

[5] C. Becchi, A. Rouet and A. Stora, Phys. Lett. B 52 (1974) 244.

[6] L. Baulieu, Phys. Rep. 129 (1985) 1. 